\title{
Rotordynamic Forces Acting on a Two-Stage Inducer
}

\author{
Naoki Nagao ${ }^{1 *}$, Katsuji Nagaura ${ }^{2}$, Tsutomu Tamura ${ }^{2}$, Satoshi Hasegawa ${ }^{3}$, Masaharu Uchiumi $^{3}$
}

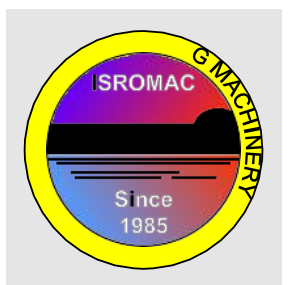

ISROMAC 2016

International

Symposium on Transport

Phenomena and

Dynamics of

Rotating Machinery

Hawaii, Honolulu

April 10-15, 2016
Abstract

Rocket turbopumps sometimes experience self-excited shaft vibration due to rotordynamic forces. To prevent this vibration, a key step is to establish a method to measure and evaluate the rotordynamic forces that act on turbopump components. In this study, we measured rotordynamic forces acting on a two-stage inducer using a rotordynamic test stand developed in 2012 at Kakuda Space Center. In noncavitating conditions, we didn't observe strong non-linearities in rotordynamic forces in the inducer at low flow-rate conditions. The results of the pressure fluctuation on the inducer showed that rotordynamic forces were mainly excited in the second stage of the inducer. In cavitating conditions, we found that there is no strong nonlinearity between cavitating rotordynamic forces and the whirling frequency ratio in the inducer. These results show the robustness of the rotordynamic forces acting on the inducer against the flow rate and cavitation.

\author{
Keywords \\ Rotordynamic forces - Inducer - cavitation \\ ${ }^{1} R \& D$ Directorate, JAXA, Tsukuba, Japan \\ 2 Japan AeroSpace Technology Foundation, Sendai, Japan \\ ${ }^{3} R \& D$ Directorate, JAXA, Kakuda, Japan \\ *Corresponding author: nagao.naoki@jaxa.jp
}

\section{INTRODUCTION}

Rocket engines should provide high power while being light weight; thus the rotational speed of engine turbopumps generally becomes higher than the first critical rotor speed. Therefore, rocket turbopumps sometimes experience selfexcited shaft vibration caused by rotordynamic forces. Because of the high power level and rotational speed, the rotational stability of rocket turbopumps is an important factor to consider for increasing the reliability of rocket engines.

Yoshida et al. measured rotordynamic forces acting on a three-bladed inducer under supersynchronous/synchronous rotating cavitation [1]. They reported that rotating cavitation generates an imbalance in the rotor and causes vibration in the shaft. The shaft vibration varies the clearance between the blade tip and the inner wall and promotes cavitation because tip clearance is factor that causes increased cavitation. The phenomenon is a problem involving hydrodynamics coupled with rotordynamics. Pasini et al. measured the rotordynamic forces of a four-bladed inducer, which vary under different operating conditions such as the flow coefficient, fluid temperature, and cavitation number [2]. Both the studies showed a strong nonlinearity of rotordynamic forces with respect to the vibration frequency due to the occurrence of cavitation.

As such, it is difficult to predict rotordynamic forces acting on a cavitating inducer because of their nonlinearity. A key step in designing better rotors and suppressing shaft vibration is to establish a method to measure the rotordynamic forces that act on a cavitating inducer. JAXA has previously developed a test stand that can measure rotordynamic forces. In this study, we describe our measurement of rotordynamic forces acting on a two-stage inducer using JAXA's rotordynamic test stand.

\section{EXPERIMENTAL APPARATUS}

JAXA developed a rotordynamic test stand (JARTS) at the Kakuda Space Center in 2012 [3] and it has been used in several types of test object experiments to investigate rotordynamic forces acting on rocket turbopump components. These experiments have tested the forces acting on components such as the impeller, inducer, seal, and damper. In this section, we provide an overview of the test stand.

\subsection{Configuration}

Figure 1 shows the design and appearance of the JARTS. It is composed of a vibration exciter, a motor, fluid lines, and a tank. A test object, e.g., an impeller or inducer, is connected vertically to the shaft of the vibration exciter which is connected vertically to an electrical motor. This means that the test object and the shaft rotate around the vertical axis. The vibration exciter controls the shaft in the radial and axial directions. Room-temperature water is stored at the tank and flows through the fluid lines by the rotation of the test object. The flow rate is measured using an electromagnetic flow meter in the fluid line. The temperature and pressure of the water are also measured. 


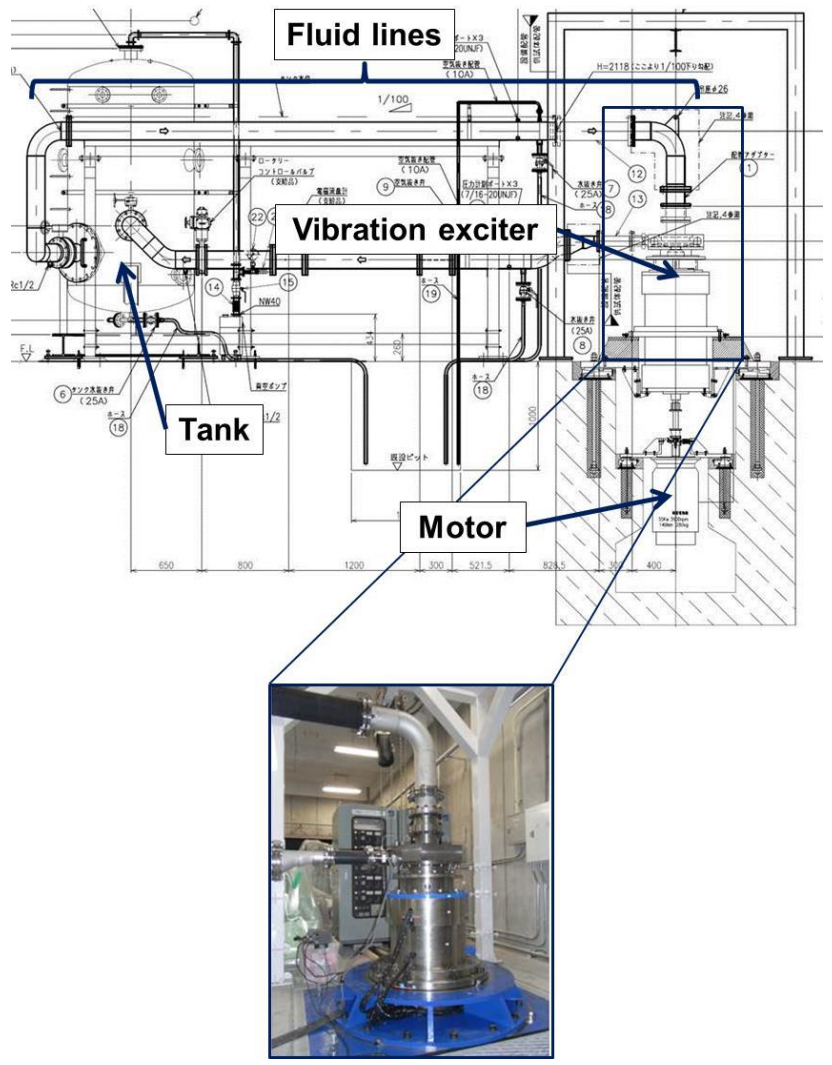

Figure 1. Appearance of the JARTS

\subsection{Measurement control system}

The turbopump shaft whirls during operation. This whirling motion generates rotordynamic forces that can cause selfexcited shaft vibrations. The rotordynamic forces are affected by the radius and frequency of the whirling. Therefore to measure rotordynamic forces acting on turbopump components, an apparatus is needed that can rotate and vibrate the shaft for any given whirling condition and then measure the magnitude and direction of the forces. The measurement control system of the JARTS vibration exciter uses magnetic bearings, similar to those used in the EBARA rotordynamics test stand [4].

Figure 2 shows a sectional view of the vibration exciter which has two radial and one axial magnetic bearings. Gap sensors measure the shaft displacement in the radial and axial directions. Figure 3 shows a diagram of the control system. Gap sensors in the $X, Y$, and $Z$ directions measure the shaft displacement. The computer calculates the coil current to keep the shaft at the target displacement. The output signal from the function generator is amplified, and the electromagnetic force occurs by a coil current in the magnetic bearings. This control system makes it possible to not only vibrate the shaft in one direction but also control the shaft in parallel and conical modes, as shown in Figure 4.

The rotordynamic forces are estimated based on the coil current in magnetic bearings. The electromagnetic force $\mathrm{F}$ generated by the magnetic bearings is calculated as follows:

$$
F=K \frac{\mu_{0} N^{2} A I^{2}}{4 g^{2}}
$$

where $K$ is the pole shape coefficient, $\mu_{0}$ is the air
Article Title -2

permeability, $\mathrm{N}$ is the number of turns of the coil, $\mathrm{A}$ is the magnetic path cross-sectional area, I is the coil current, and $g$ is the air gap. Using Equation 1, we can determine the electromagnetic force generated by the magnetic bearings based on the coil current when the shaft displacement is negligible small against the air gap. A fluid force containing a rotordynamic force and a steady force which occurs due to asymmetry of the fluid or of the geometry of the casing is the difference between an electromagnetic force needed to whirl the shaft in the fluid and that needed to whirl the shaft in air.

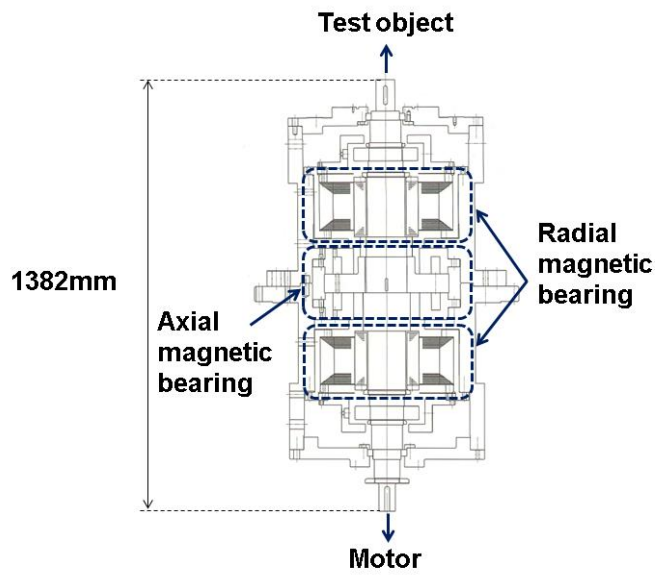

Figure 2. Magnetic bearings in the vibration exciter

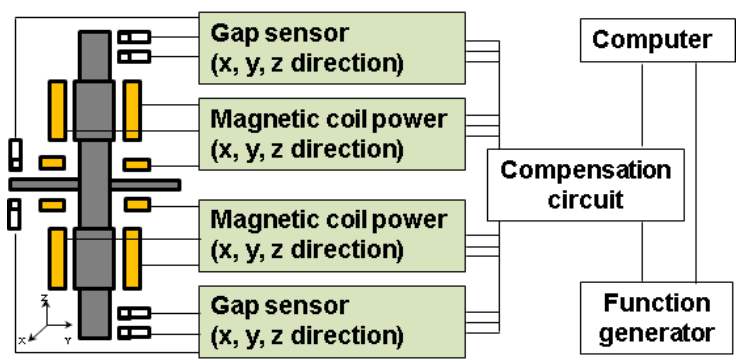

Figure 3. Control system diagram

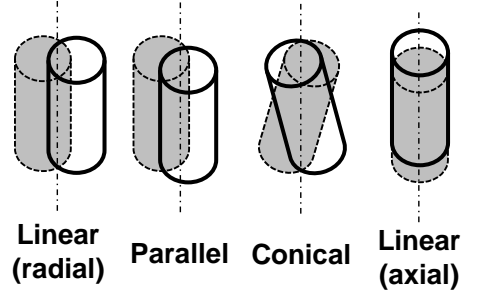

Figure 4. Vibration modes

\section{TEST OBJECT}

In this study, the test object is a two-stage inducer, as shown in the photograph in Figure 5. It has 3 blades in the first stage and 9 blades in the second. The outer diameter is $160 \mathrm{~mm}$. Figure 6 shows a sectional view of the test object assembly installed in the vibration exciter. An acrylic casing was used to visually observe the cavitation process. The inducer has 4 fluctuation pressure sensors that make it possible to measure the pressure fluctuation at the inlet of the first stage, middle of the first stage, inlet of the second stage and outlet of the second stage. 


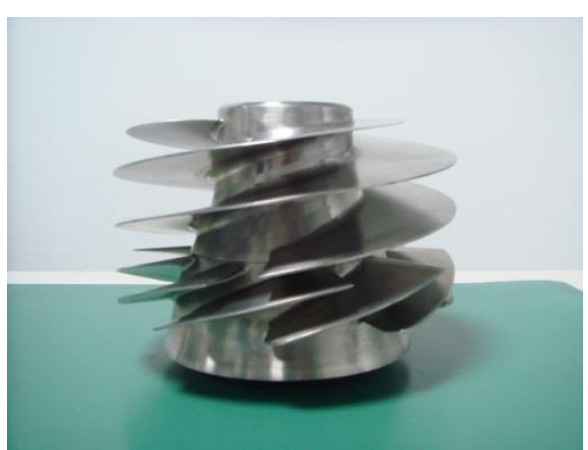

Figure 5. Two-stage inducer

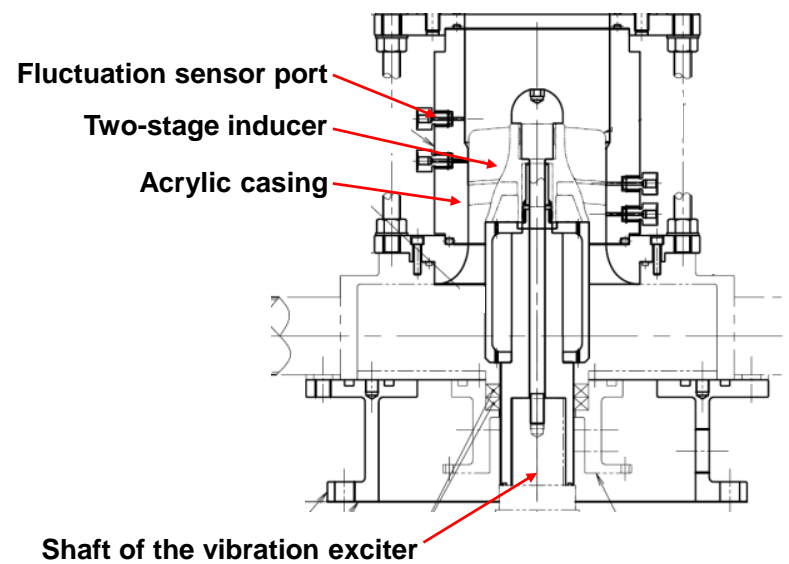

Figure 6. Four fluctuation sensor ports on the Acrylic casing

\section{EVALUATION METHOD}

Figure 7 shows a definition of the rotordynamic forces. The shaft rotates at $\Omega$ and whirls at $\omega$. The whirling radius is $\varepsilon$. The rotordynamic forces are decomposed into a force normal to the direction of the whirling motion " $F_{n}$ " (radius outward direction is positive) and a force in the direction of the forward whirling motion " $F_{t}$ " (the direction, same as that of rotation, is positive). These two forces are generally presented in the dimensionless form as functions of the whirl frequency ratio, " $\omega / \Omega$ ", as follows:

$$
\begin{gathered}
f_{n}=M(\omega / \Omega)^{2}-c(\omega / \Omega)-K \\
f_{t}=-m(\omega / \Omega)^{2}-C(\omega / \Omega)+k
\end{gathered}
$$

where the forces are normalized as

$$
f_{n, t}=F_{n, t} /\left(\rho \pi R_{2}{ }^{2} b_{2} \varepsilon \Omega^{2}\right)
$$

where " $\rho$ " is the fluid density, " $R_{2}$ " is the outlet radius of the inducer, and " $\mathrm{b}_{2}$ " is the outlet blade height of the inducer. " $\mathrm{M}$ ", "m," "C," "c," "K," and "k" are rotordynamic coefficients.

In the range of " $\omega / \Omega$ " $>0$, a positive tangential fluid force $\left(F_{t}\right.$ $>0$ ) has a destabilizing effect because it promotes a whirling motion in the shaft. In the same manner, a positive normal fluid force $\left(F_{n}>0\right)$ has an inertia effect. However in a general turbopump, the radial stiffness of the bearings is sufficiently large and thus does not negatively affect the turbopump system.

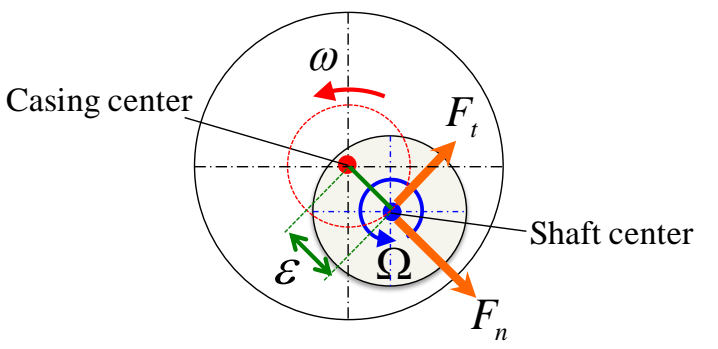

Rotordynamic force effect at $\omega / \Omega>0$

$$
\begin{aligned}
& F_{n}>0: \text { inertia effect } \\
& F_{n}<0: \text { restoring effect } \\
& F_{t}>0: \text { destabilizing effect } \\
& F_{t}<0: \text { damping effect }
\end{aligned}
$$

Figure 7. Definition of the rotordynamic forces

\section{RESULTS AND DISCUSSION}

The characteristics of the inducer are described in this section, including the performance curve at noncavitating condition, noncavitating rotordynamic forces, and cavitating rotordynamic forces.

\subsection{Performance curve at noncavitating condition}

Figure 8 shows the performance curve at noncavitation when the rotation speed is $2000 \mathrm{rpm}$ without whirling and the fluid is water at room temperature. The vertical axis is the inducer head coefficient normalized by the reference head coefficient, " $\Psi_{n}$," when the flow rate, " $Q$," is the reference flow rate, " $Q_{n}$," and the horizontal axis is the flow rate normalized by the reference flow rate. " $\Psi 1$," is the head coefficient of the first stage defined by the pressure difference between the inlet and outlet of the first stage. " $\Psi 2$ " is the head coefficient of the second stage defined by the pressure difference between the inlet and outlet of the second stage. " $\Psi$ " is the total head coefficient of the first and second stages.

$\Psi 1$ decreases against the flow rate, and $\Psi 2$ increases with the flow rate. At the reference point, $\Psi 1$ and $\Psi 2$ become almost identical. $\Psi$ decreases at $Q / Q_{n}>0.5$. With respect to the instability phenomena, for example, the rotating stall did not occur within the range measured, and the inducer worked well over a wide range. 


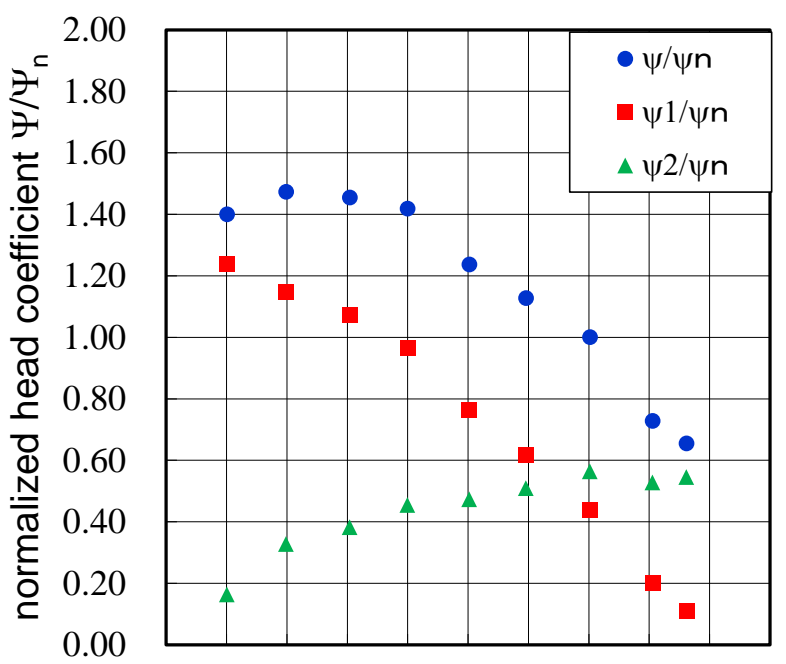

$\begin{array}{lllllllllll}0.3 & 0.4 & 0.5 & 0.6 & 0.7 & 0.8 & 0.9 & 1.0 & 1.1 & 1.2 & 1.3\end{array}$ normalized flow rate $Q / Q_{n}$

Figure 8. Performance curve at noncavitating condition

\subsection{Noncavitating rotordynamic forces}

We conducted a series of noncavitating rotordynamic force tests by whirling the inducer with the JARTS vibration exciter. In addition to measuring the rotordynamic forces, we measured the pressure fluctuation around the inducer in four positions to clarify where the forces mainly occur. Figure 9 shows the experimental results of the dimensionless normal rotordynamic forces $f_{n}$ and the dimensionless tangential rotordynamic force $f_{t}$ as functions of the whirl frequency ratio, $\omega / \Omega$. The test conditions are as follows: the rotation speed was $2000 \mathrm{rpm}$, whirling radius was $100 \mu \mathrm{m}$ and flow-rate ratio ranged from 0.6 to 1.16. The results show both $f_{n}$ and $f_{t}$ have positive values over the entire range of the whirl frequency ratio. This means that $f_{n}$ has an inertia effect and $f_{t}$ has a destabilizing effect at $\omega / \Omega>0$. Interestingly, the shapes of $f_{n}$ and $f_{t}$ of the inducer are slightly similar to those of a typical shrouded impeller [5]; that is, the shape of $f_{n}$ is a quadratic curve that has a vertex at $\omega / \Omega>0$ and the shape of $f_{t}$ is a negatively inclined line. But to be exact, the results show $f_{n}$ is not a perfect quadratic curve at positive whirl ratio and $f_{t}$ is roughly linear. Arndt, N. and Franz, R. reported that in axial-flow inducers, as the flow coefficient decreases, somewhat strong non-linearities begin to appear in the values of $f_{t}$ and to a lesser degree in $f_{n}$ at small and positive whirl frequency ratios[6]. However we didn't observe strong non-linearities in the $f_{n}$ and $f_{t}$ values of the two-stage inducer at low flow-rate ratios. This result shows that the effect of a flow-rate ratio on the rotordynamic forces acting on the inducer is small. In other words, the inducer has rotordynamic force robustness against flow-rates over a wide range.

Figure 10 shows the FFT analysis results for the pressure fluctuations on the inducer. The rotation speed was 2000 rpm $(33 \mathrm{~Hz})$, flow rate ratio was 1.0, whirling radius was $100 \mu \mathrm{m}$, and the whirling frequency was $40 \mathrm{~Hz}$ in the negative direction. The amplitude of the pressure fluctuation at the $40 \mathrm{~Hz}$ of inlet and outlet of the second stage is much larger than that of inlet and middle of the first stage. The results show that the rotordynamic forces mainly occur in the second stage. We consider that this is because the tip clearance in the second stage is smaller than that in the first stage. As such, the pressure fluctuation in the second stage is larger than that in the first stage. The tip clearance in the first stage is $780 \mu \mathrm{m}$ and that at second stage is $500 \mu \mathrm{m}$.

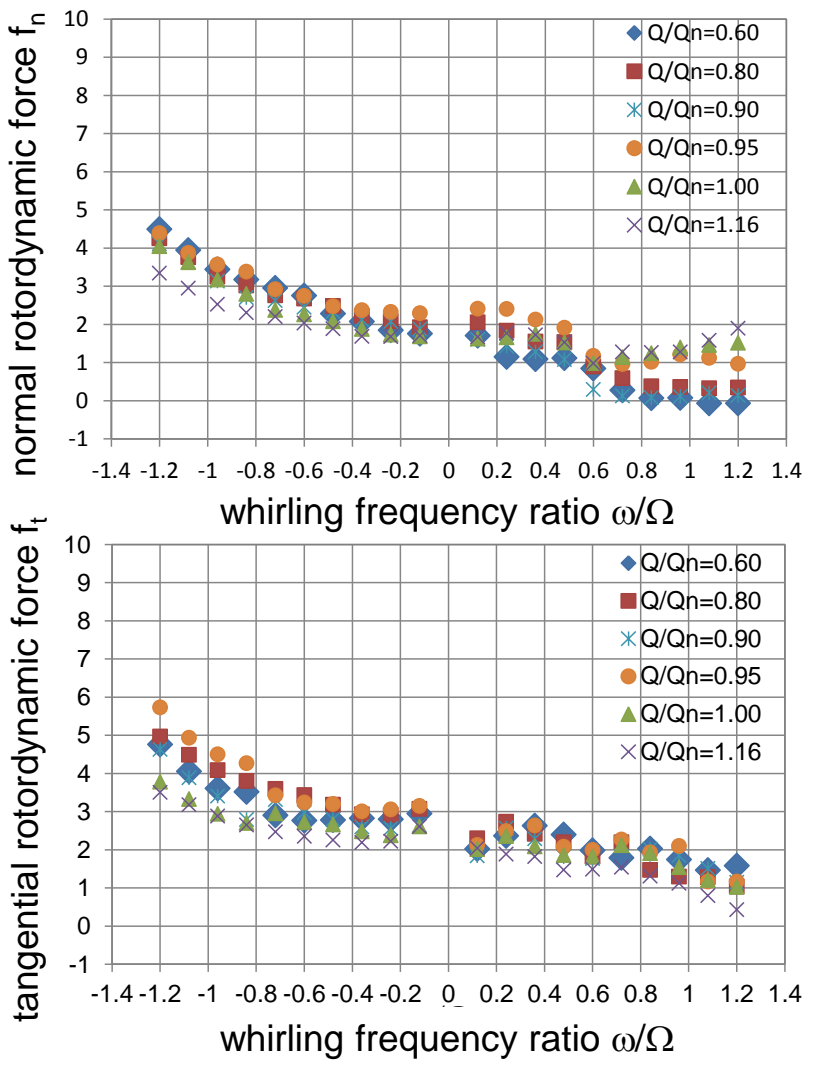

Figure 9. Characteristics of $f_{n}$ and $f_{t}$ related to varied $Q / Q_{n}$ 

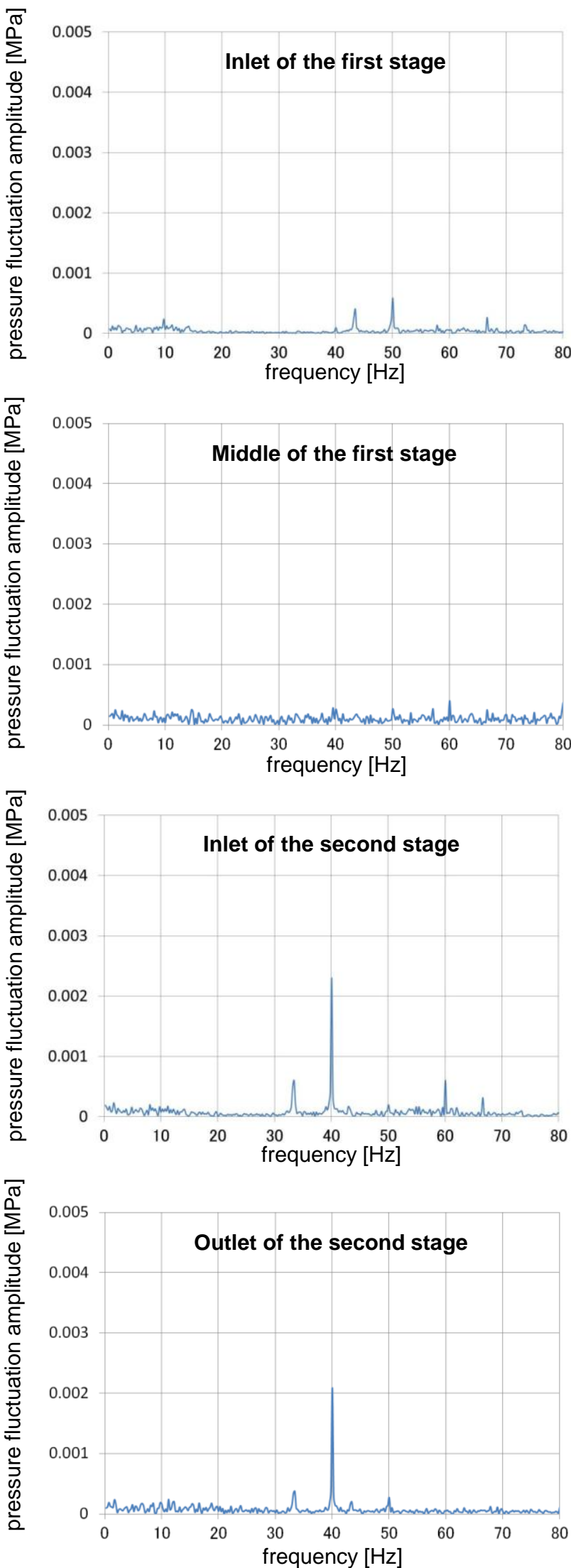

Figure 10. FFT analyses of pressure fluctuation on inducer

\subsection{Cavitating rotordynamic forces}

We conducted a series of cavitating rotordynamic force tests by changing the inlet pressure to investigate the effect of the cavitation number " $\sigma$ " on the rotordynamic forces. We changed the cavitation number from 0.127 to 0.013 . Figure 11 shows photograph of the cavitation around the inducer related to cavitation number. No cavitation occurred at $\sigma=0.127$. Tip vortex cavitation began to occur at $\sigma=0.042$. Tip vortex cavitation occurred all around the blades at $\sigma=0.013$. Figure 12 shows the characteristics of $f_{n}$ and $f_{t}$ related to cavitation number. The test conditions are as follows: the rotation speed was $3000 \mathrm{rpm}$, whirling radius was $50 \mu \mathrm{m}$, flowrate ratio was 1.0 , and cavitation numbers were 0.013 , 0.042 , and 0.127 . In past discussions of the rotordynamic forces acting on a cavitating inducer [1] [2], the forces have shown strong nonlinearity against the whirl frequency ratio because of the occurrence of cavitation. However, the results from our two-stage inducer test show that while nonlinearity in $f_{n}$ is observed at $\omega / \Omega=0.6$, it occurs to a much smaller degree than in past studies. When the absolute value of the whirling frequency ratio is high, $f_{n}$ becomes higher based on the decrease in the cavitation number. $f_{t}$ shows no change with the cavitation number. The inducer was designed to improve cavitation instability. As designed, no obvious cavitation instability occurred at the experimented cavitation numbers. Therefore the rotordynamic forces showed no strong nonlinearity or sensitivity to the cavitation number.
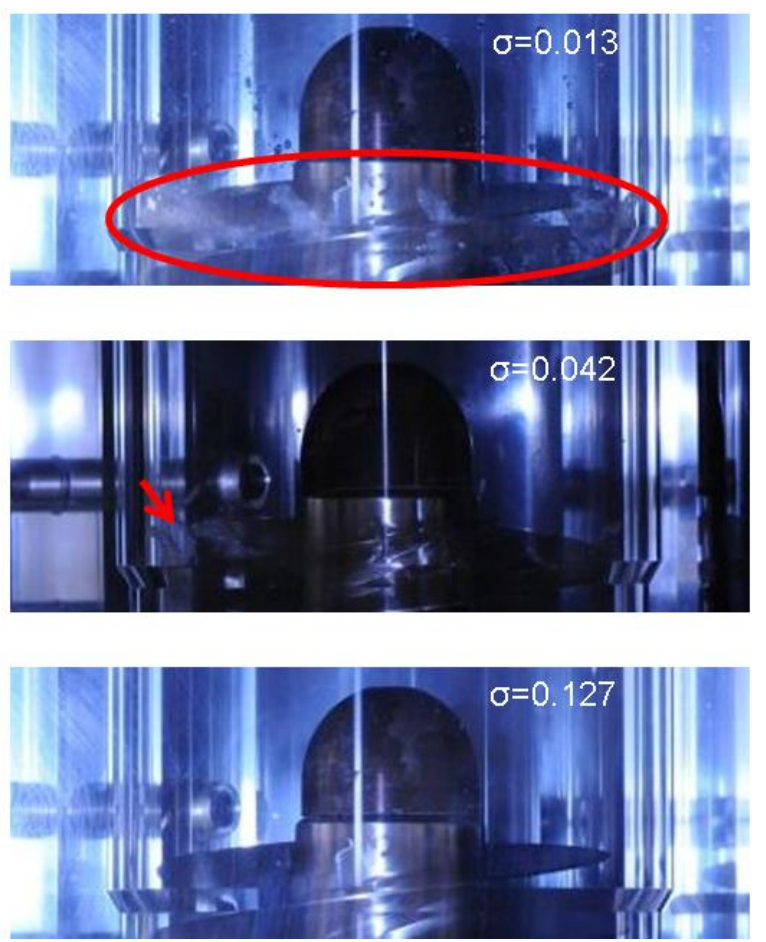

Figure 11. Cavitation around the inducer related to cavitation number 


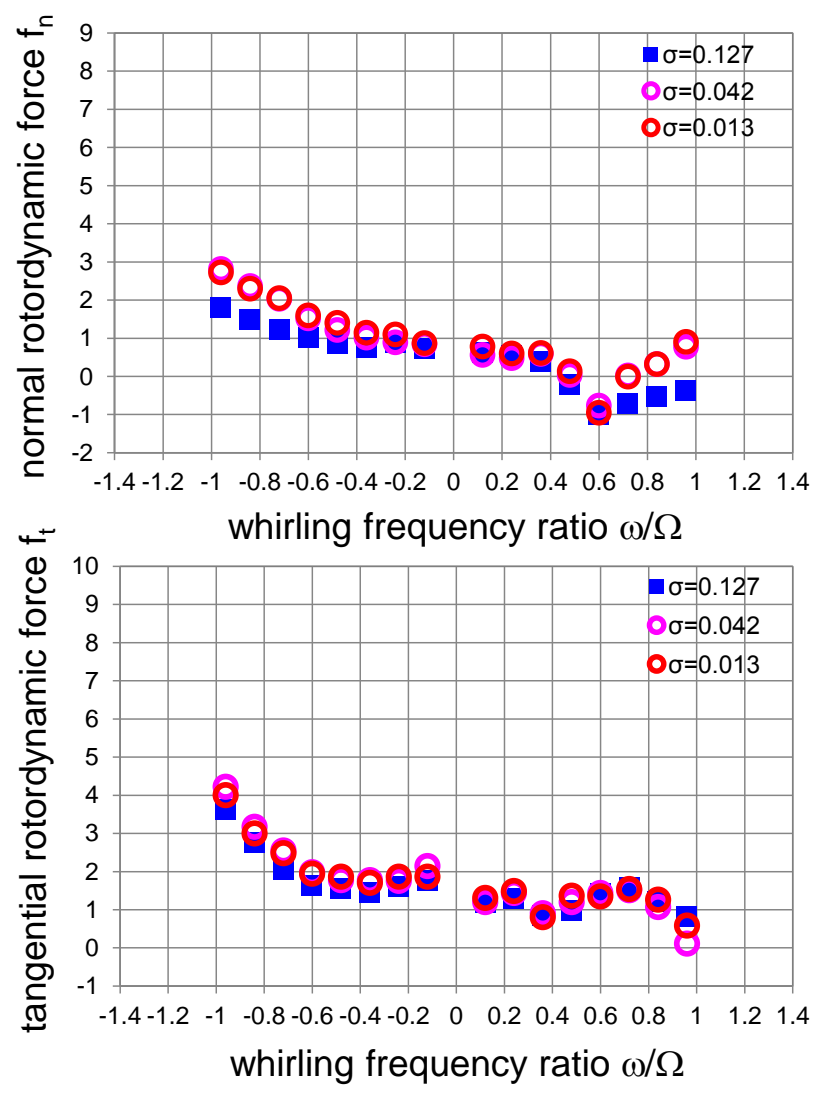

Figure 12. Characteristics of $f_{n}$ and $f_{t}$ related to cavitation number

\section{Conclusions}

In this study, we measured the rotordynamic forces acting on a two-stage inducer under noncavitating and cavitating conditions using a rotordynamic test stand controlled by magnetic bearings. The experimental results and discussion are summarized as follows:

(1) Unlike past studies, we didn't observe strong non-linearities in the normal rotordynamic force " $\mathrm{f}_{\mathrm{n}}$ " and tangential rotordynamic force " $\mathrm{f}_{\mathrm{t}}$ " of the inducer at a low flow-rate ratio without cavitation. The result shows that the affect of a flow-rate ratio on the rotordynamic forces acting on the inducer is small.

(2) The results of pressure fluctuation show that noncavitating rotordynamic forces mainly occur in the second stage because the tip clearance in the second stage is smaller than that in the first stage.

(3) We didn't observe strong nonlinearity of the cavitating rotordynamic forces against the whirl frequency ratio in the inducer.

\section{ACKNOWLEDGMENTS}

Our heartfelt appreciation goes to Dr. Yoshida who conducted these experiments with us when he was working at JAXA and who provided insightful comments and suggestions. We would like to offer our special thanks to Dr. Eguchi, who developed JARTS and offered us constructive comments and warm encouragement for this test campaign.

\section{REFERENCES}

[1] Yoshida, Y., Eguchi, M., Motomura, T., Uchiumi, M., Kure, H., and Maruta, Y., 2010, "Rotordynamic Forces Acting on Three-Bladed Inducer Under Supersynchronous/Synchronous Rotating Cavitation." ASME Journal of Fluids Engineering, June 2010, Vol.132

[2] Pasini, A., Torre, L., Cervone, A., and d'Agostino, L., 2010, "Rotordynamic Forces on a Four Bladed Inducer" $46^{\text {th }}$ AIAA/ASME/SAE/ASEE Joint Propulsion Conference \& Exhibit 25-28 July 2010, Nashville, TN

[3] Nagao, N., Eguchi, M., Uchiumi, M., and Yoshida, Y., 2012, "Measurement of Rotordynamic Forces on a Turbopump“ Journal of TSJ, 'Turbomachinery' July 2012, Vol.40-7

[4] Eguchi, M., and Maruta, Y., 2003, "Development of Rotordynamics Measurement System with Active Magnetic Bearings," Proc. 10th Asia-Pacific Vibration Conference, Gold Coast, Australia, Vol.1, pp.115-120.

[5] Brennen, C.E., 1994, "Hydrodynamics of Pumps," Oxford University Press.

[6] Arndt, N. and Franz, R., 1986, "Observations of hydrodynamic forces on several inducers including the SSME LPOTP," Calif. Inst. of Tech., Div. Eng. and Appl. Sci., Report No. E249.3. 